\title{
Two Cases of Complications during Percutaneous Coronary Intervention for Myocardial Infarction in Patients with Concurrent Chronic Total Occlusion in an Emergency Setting
}

\author{
Donghoon Han', Sehun Kim', Sang Jun Park², Jae Hyuk Choi'
}

Departments of ${ }^{1}$ Internal Medicine and ${ }^{2}$ Cardiothoracic Surgery, Cardiovascular Center, Hallym University Hangang Sacred Heart Hospital, Seoul, Korea

\begin{abstract}
Morbidity and mortality rates associated with acute myocardial infarction accompanying chronic total occlusion are comparatively high. European guidelines recommend primary intervention for the causative lesion in patient with acute myocardial infarction. Therefore, it is important to identify the culprit lesion. We report two cases of myocardial infarction with concurrent chronic total occlusion in an emergency setting.
\end{abstract}

Keywords: Myocardial infarction; Percutaneous coronary intervention; Culprit; Concurrent chronic total occlusion; Emergency setting

\section{INTRODUCTION}

Chronic total occlusion (CTO) is defined as $100 \%$ occlusion with a thrombolysis in myocardial infarction (TIMI) score of 0 for flow for at least 3 months [1]. CTO in acute myocardial infarction (AMI) has poor outcomes despite successful primary percutaneous coronary intervention (PCI) [2,3]. Although the pathophysiology of AMI and of CTO is different, it may be challenging to identify the culprit lesion in patients with multivessel diseases, particularly in an emergency situation [4]. We describe two cases of emergency PCI using different approaches that resulted in complications in patients with AMI and concurrent CTO.

\section{CASE REPORTS}

\section{Case 1}

A 76-year-old man was brought to the emergency department (ED) while undergoing cardiopulmonary resuscitation (CPR) due to cardiac arrest. The patient had hypertension, diabetes, and stage 5 chronic kidney disease (estimated glomerular filtration rate, 13 $\mathrm{mL} / \mathrm{min}$ ). He used aspirin, clopidogrel, an angiotensin-converting enzyme inhibitor (perindopril $4 \mathrm{mg}$ ), a beta-blocker (carvedilol 25 $\mathrm{mg}$ ), and oral hypoglycemic agents (linagliptin $5 \mathrm{mg}$ and glimepiride $4 \mathrm{mg}$ ). Before arriving at the ED, the patient reported chest pain and dyspnea. He then lost consciousness. His initial troponin I level had increased to $0.28 \mu \mathrm{g} / \mathrm{L}$. After CPR for 12 minutes in the ED, the patient achieved return of spontaneous circulation (ROSC). After ROSC, his electrocardiogram (EKG) showed T-wave inversion at the anterior leads (Fig. 1A). The TIMI risk score was 9, and we decided to perform emergency coronary angiography (CAG) based on the suspicion of AMI as the cause of the arrest. However, because his family members strongly opposed CAG and signed a 'do not resuscitate' (DNR) document, the patient was admitted to the intensive care unit without undergoing CAG. The next day, the patient remained unconscious, but his vital signs were stable. However, his troponin I level had increased to $5.02 \mu \mathrm{g} / \mathrm{L}$, and the echocardiogram showed regional wall motion abnormalities from the base to the mid inferior wall with reduced systolic function (ejection fraction, 45\%). At that time, his family members changed their previous opinion and demanded CAG. Consequently, we performed CAG, which showed three-vessel disease with $80 \%-$ $90 \%$ diffuse stenosis in the left anterior descending artery (LAD), 


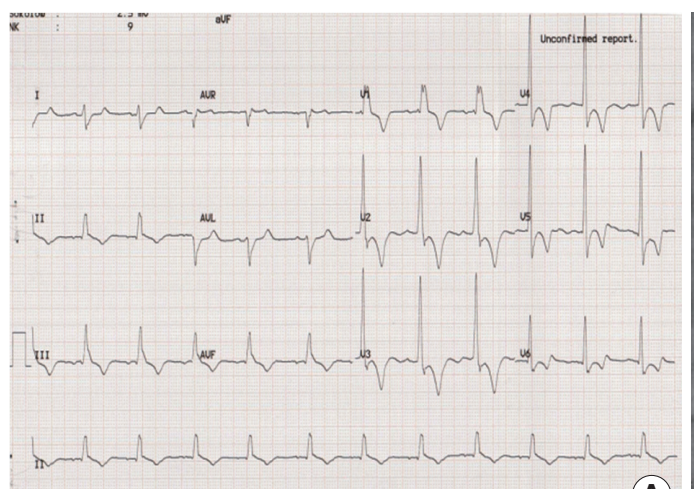

(A)
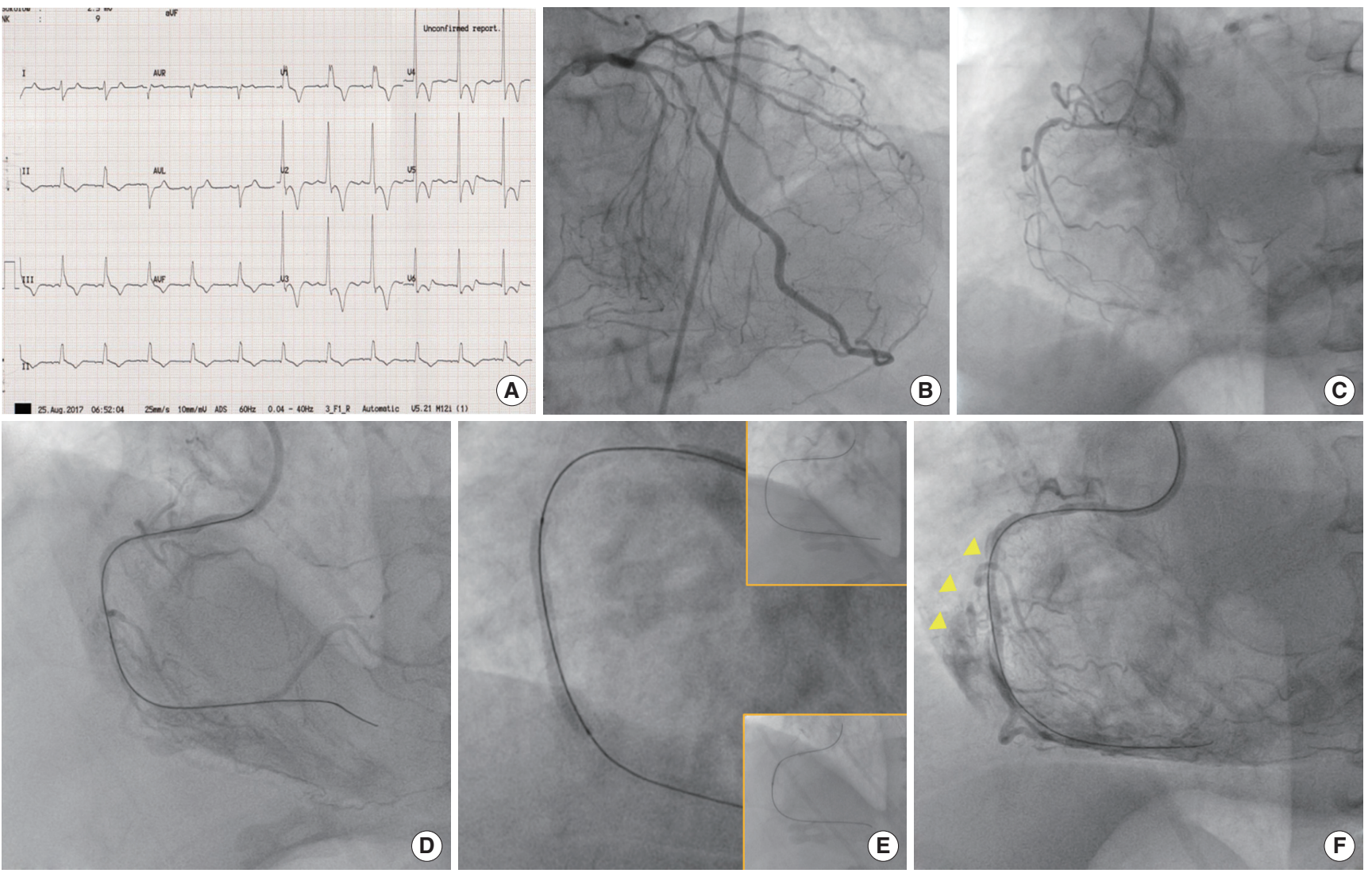

Fig. 1. (A) Electrocardiogram showed T-wave inversion in the whole precordial area. (B) Coronary angiogram showed three-vessel disease with diffuse stenosis in the $L A D$, diffuse near-total stenosis at the distal circumflex artery, and collateral flow from the LAD to the RCA. (C) The RCA showed stenosis at the proximal part, and mid-RCA chronic total occlusion was observed. (D) A wire and microcatheter were successfully advanced to the posterior lateral branch. (E) A stent was implanted. (F) After stenting, leakage of contrast was observed in the proximal part of the stent (yellow arrowheads). LAD, left anterior descending artery; RCA, right coronary artery.

diffuse near-total stenosis at the distal circumflex artery, and 70\% tubular stenosis at the proximal right coronary artery (RCA). In addition, mid-RCA CTO with collateral flow from the distal LAD and septal branch was observed (Fig. 1B, C). Because the patient's vital signs remained stable during CAG, we decided to treat the RCA CTO rather than the feeding vessel (the LAD). A wire (Gaia 1st; Asahi Intecc Co. Ltd., Tokyo, Japan) was loaded into a microcatheter (Corsair, Asahi Intecc Co. Ltd.) and passed through the proximal cap of the CTO lesion; however, it failed to penetrate the distal cap. Subsequently, we changed the wire to a Gaia 2nd wire (Asahi Intecc Co. Ltd.), which was successfully passed (Fig. 1D). Thereafter, predilatation was repeatedly performed with a $1.0 \times$ 6-mm balloon with 14 atm of pressure, followed by sequential ballooning with a $2.0 \times 15-\mathrm{mm}$ balloon with nominal pressure. Afterward, the blood flow was recovered. Consequently, we implanted a $2.75 \times 32-\mathrm{mm}$ stent (SYNERGY; Boston Scientific, Marlborough, MA, USA) (Fig. 1E). However, contrast leakage was ob- served in the proximal part of the stent, and the patient experienced cardiac arrest (Fig. 1F). All procedures were stopped and 1 $\mathrm{mg}$ of epinephrine and $0.5 \mathrm{mg}$ of atropine were administered intravenously during CPR. After 30 seconds of CPR, the patient achieved ROSC and was returned to the intensive care unit. There was no covered stent in the angiography room at that time; therefore, we focused on stabilizing his vital signs instead of prolonging the procedure time. The patient's family members already agreed with the DNR order; therefore, we did not recommend any other procedures such as pericardiocentesis or bypass surgery. The patient died 4 days after the procedure.

\section{Case 2}

A 56-year-old man visited the ED and reported chest pain. He had hypertension and diabetes and used aspirin. He had experienced dyspnea 3 days previously, but he did not visit the hospital at that time. The dyspnea became worse, and new chest pain oc- 

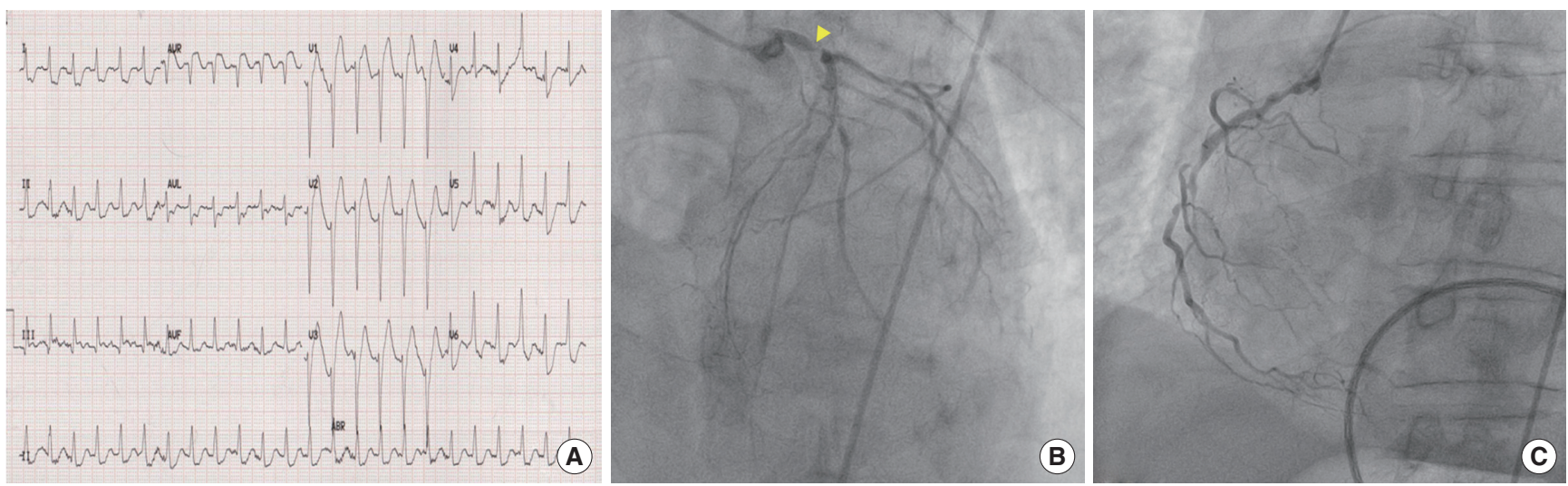

Fig. 2. (A) Electrocardiogram showed ST segment elevation at the aVR and V1-3 leads and reciprocal changes at the lateral leads. (B) The coronary angiogram showed tight stenosis with heavy calcification at the distal left main coronary artery (yellow arrow) and diffuse disease at the mid-to-distal LAD. (C) Tubular stenosis at the proximal RCA and chronic total occlusion at the mid-RCA were observed. (D) After predilatation (orange box), the lesion remained tight. (E) We attempted to advance the stent; however, the guiding catheter pushed back because of poor support (orange box). Subsequently, ventricular fibrillation suddenly occurred, and the stent was retrieved. LAD, left anterior descending artery; RCA, right coronary artery.
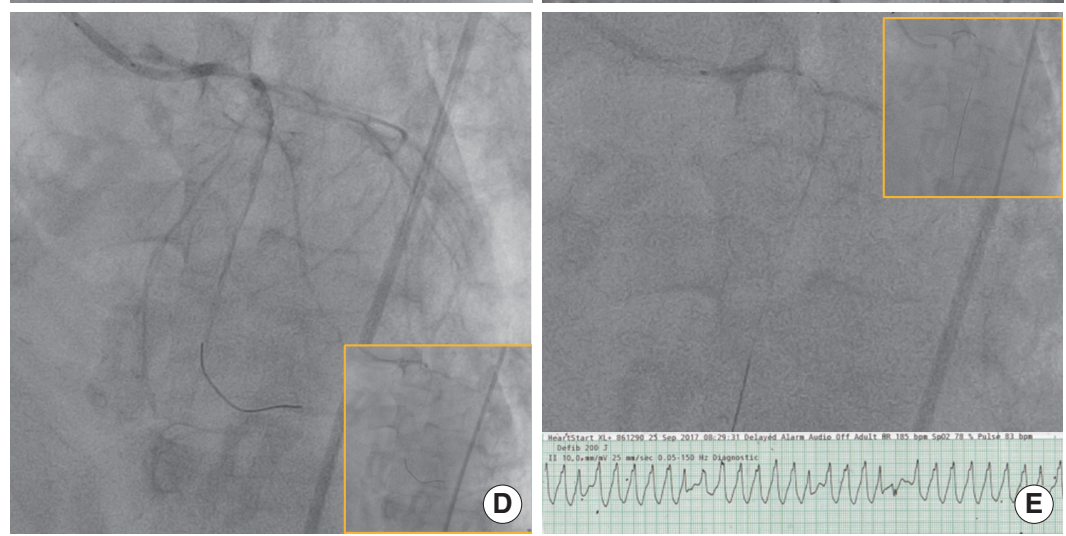

curred 6 hours before visiting the ED. On admission, the EKG showed ST segment elevation at the aVR and V1-3 leads and reciprocal changes at the lateral leads (Fig. 2A). The patient's TIMI risk score was 5 . We performed emergency CAG for ST-segment elevation MI. The emergency coronary angiogram revealed tight stenosis with heavy calcification at the distal left main coronary artery and proximal LAD, diffuse disease at the mid-to-distal LAD, tubular stenosis of $80 \%$ at the proximal RCA, and CTO at the midRCA, with collateral flow from the LAD (Fig. 2B, C). Because of the EKG findings, we inserted a wire (Runthrough NS; Terumo Interventional Systems, Somerset, NJ, USA) in the LAD after engaging the guiding catheter. Even though only one wire was inserted, the blood flow became very slow. Although the flow slightly recovered to nominal pressure after rapid ballooning with a 2.0 $\times 15-\mathrm{mm}$ balloon, the dilatation was not enough to implant a stent because of heavy calcification (Fig. 2D). Suddenly, the patient's blood pressure decreased; therefore, we immediately administered norepinephrine and dopamine. The patient's vital signs became stable with inotropes, and we tried to insert a stent. However, the guiding catheter was pushed back because of poor support, and we inserted another wire (Sion; Asahi Intecc Co.
Ltd.) to acquire greater back-up force. Nonetheless, we could not pass the stent through the lesion (Fig. 2E). Subsequently, ventricular fibrillation suddenly occurred due to flow disturbance created by the stent. We immediately retrieved the stent and started CPR (Fig. 2E). After ROSC, we used an extra backup 3.5 guiding catheter and performed ballooning of the lesion with a $2.5 \times 15-\mathrm{mm}$ balloon. We then successfully inserted the stent in the lesion. However, afterward, a second arrest suddenly occurred. The cardiothoracic surgery team was called to start extracorporeal membrane oxygenation (ECMO). After performing and controlling ECMO completely, we recommended emergency coronary artery bypass grafting (CABG) because the patient's condition was unstable and arrest occurred 2 times during the procedure. After emergency $\mathrm{CABG}$, the ECMO catheters were removed on postoperative day 4 . The patient was still alive at the time of writing this article.

\section{DISCUSSION}

We reviewed two patients with a similar coronary artery condition treated using different approaches. As we have shown, ap- 
proaching the culprit lesion associated with AMI and using a team approach are important for determining the prognosis in emergency situations. In case 1 , we should have approached the LAD lesion, which was the suspected culprit lesion, instead of the CTO. In case 2, applying mechanical circulatory support and performing emergency surgery contributed to better results.

CTO can arise from thrombotic occlusion of vessels, subsequent thrombus organization, and tissue remodeling [5]. However, the majority of patients with CTO rarely have a history of myocardial infarction, and the prevalence of CTO among patients undergoing cardiac catheterization has been reported to range from $18 \%$ to $54 \%$ [6-8]. Based on the severity of symptoms, concomitant coronary artery disease (CAD), ischemic burden, and patient risk profiles, different treatment options may be chosen for each patient. However, regardless of the presence of CTO, patients with CAD should be counseled about the risks and undergo aggressive medical therapy for CAD. Medical therapy is one option for patients with no symptoms or mild symptoms. For patients with a large ischemic burden or refractory symptoms despite optimal medical therapy, revascularization should be considered. CABG should be considered for patients with concomitant multivessel or left main $\mathrm{CAD}$, or for those requiring cardiac surgery for other reasons. Patients with CTO are still more likely to be referred for CABG than PCI. However, with the development of new devices and techniques, a higher success rate with PCI for CTO lesions and fewer complications related to the procedure are expected [6,9]. Moreover, with the development of mechanical circulatory support, such as the intra-aortic balloon pump and ECMO, treatment can be performed for those with multivessel disease or LV dysfunction and during unstable situations that may occur during procedures [10]. Consequently, in the emergency setting, as in our cases, the treatment strategy is mainly based on the physician's judgment.

In conclusion, it is necessary to develop a more careful approach to diagnosing the culprit lesion in patients with multivessel disease, including CTO, who present with myocardial infarction. During unstable situations, clinicians should not hesitate to use mechanical circulatory support. These patients may be candidates for $\mathrm{CABG}$; however, there may be differences in the proficiency levels of physicians who perform PCI. Therefore, physicians should use caution when deciding when and how to treat patients similar to those reported here.

\section{ACKNOWLEDGMENTS}

We extend our thanks to the patients and members of their families.

\section{REFERENCES}

1. Brilakis ES. Manual of coronary chronic total occlusion interventions: a step-by-step approach. 2nd ed. London: Elsevier; 2017.

2. Van der Schaaf RJ, Timmer JR, Ottervanger JP, Hoorntje JC, de Boer MJ, Suryapranata $\mathrm{H}$, et al. Long-term impact of multivessel disease on causespecific mortality after ST elevation myocardial infarction treated with reperfusion therapy. Heart 2006;92:1760-3.

3. Tajstra M, Gasior M, Gierlotka M, Pres D, Hawranek M, Trzeciak P, et al. Comparison of five-year outcomes of patients with and without chronic total occlusion of noninfarct coronary artery after primary coronary intervention for ST-segment elevation acute myocardial infarction. Am J Cardiol 2012;109:208-13.

4. Kang J, Jeon KH, Kim SW, Park JJ, Yoon CH, Suh JW, et al. Evolution of nonculprit coronary atherosclerotic plaques assessed by serial virtual histology intravascular ultrasound in patients with ST-segment elevation myocardial infarction and chronic total occlusion. Coron Artery Dis 2016; 27:650-7.

5. Katsuragawa M, Fujiwara H, Miyamae M, Sasayama S. Histologic studies in percutaneous transluminal coronary angioplasty for chronic total occlusion: comparison of tapering and abrupt types of occlusion and short and long occluded segments. J Am Coll Cardiol 1993;21:604-11.

6. Jeroudi OM, Alomar ME, Michael TT, El Sabbagh A, Patel VG, Mogabgab $\mathrm{O}$, et al. Prevalence and management of coronary chronic total occlusions in a tertiary Veterans Affairs hospital. Catheter Cardiovasc Interv 2014;84:637-43.

7. Fefer P, Knudtson ML, Cheema AN, Galbraith PD, Osherov AB, Yalonetsky $S$, et al. Current perspectives on coronary chronic total occlusions: the Canadian Multicenter Chronic Total Occlusions Registry. J Am Coll Cardiol 2012;59:991-7.

8. Christofferson RD, Lehmann KG, Martin GV, Every N, Caldwell JH, Kapadia SR. Effect of chronic total coronary occlusion on treatment strategy. Am J Cardiol 2005;95:1088-91.

9. Grantham JA, Marso SP, Spertus J, House J, Holmes DR Jr, Rutherford BD. Chronic total occlusion angioplasty in the United States. JACC Cardiovasc Interv 2009;2:479-86.

10. Mandawat A, Rao SV. Percutaneous mechanical circulatory support devices in cardiogenic shock. Circ Cardiovasc Interv 2017;10:e004337. 